\title{
Haptic feedback in the da Vinci Research Kit (dVRK): A user study based on grasping, palpation, and incision tasks
}

\author{
Arianna Saracino ${ }^{1,2}$ | Anton Deguet ${ }^{3}$ | Fabio Staderini ${ }^{4}$ | Mohamed Nassim Boushaki ${ }^{1}$ | \\ Fabio Cianchi ${ }^{4}$ | Arianna Menciassi ${ }^{1}$ | Edoardo Sinibaldi ${ }^{2}$
}

\author{
${ }^{1}$ The BioRobotics Institute, Scuola Superiore \\ Sant'Anna, Pontedera, Italy \\ ${ }^{2}$ Center for Micro-BioRobotics, Istituto \\ Italiano di Tecnologia, Pontedera, Italy \\ ${ }^{3}$ Laboratory for Computational Sensing and \\ Robotics, Johns Hopkins University, Baltimore, \\ Maryland \\ ${ }^{4}$ Center of Oncological Minimally Invasive \\ Surgery, Department of Surgery and \\ Translational Medicine, University of Florence, \\ Florence, Italy

\section{Correspondence} \\ Arianna Menciassi, The BioRobotics Institute, \\ Scuola Superiore Sant'Anna, Viale Rinaldo \\ Piaggio 34, 56025 Pontedera, Italy. \\ Email: arianna.menciassi@santannapisa.it \\ Edoardo Sinibaldi, Center for Micro- \\ BioRobotics, Istituto Italiano di Tecnologia, \\ Viale Rinaldo Piaggio 34, 56025 Pontedera, \\ Italy. \\ Email: edoardo.sinibaldi@iit.it
}

Funding information

Regione Toscana

\begin{abstract}
Background: It was suggested that the lack of haptic feedback, formerly considered a limitation for the da Vinci robotic system, does not affect robotic surgeons because of training and compensation based on visual feedback. However, conclusive studies are still missing, and the interest in force reflection is rising again.

Methods: We integrated a seven-DoF master into the da Vinci Research Kit. We designed tissue grasping, palpation, and incision tasks with robotic surgeons, to be performed by three groups of users (expert surgeons, medical residents, and nonsurgeons, five users/group), either with or without haptic feedback. Taskspecific quantitative metrics and a questionnaire were used for assessment.
\end{abstract}

Results: Force reflection made a statistically significant difference for both palpation (improved inclusion detection rate) and incision (decreased tissue damage).

Conclusions: Haptic feedback can improve key surgical outcomes for tasks requiring a pronounced cognitive burden for the surgeon, to be possibly negotiated with longer completion times.

\section{1 | INTRODUCTION}

Haptic feedback holds the promise to restore the sense of touch in applications ranging from human-robot interaction and gaming to surgery and training. Kinesthetic (force/joint-related) and tactile (cutaneous/skin-related) sensations are both closely connected to the concept of haptic feeling, as two sides of the same coin. ${ }^{1}$ Conveying such a range of additional information to the human end-user increases their sense of telepresence at the remote site. ${ }^{2}$ The restoration of haptic feedback, therefore, is supposed to improve accuracy and safety. ${ }^{3}$ As a matter of fact, haptics in robot-assisted surgery received a growing interest in the last decades. ${ }^{4}$

A loss of haptic sensation occurred when minimally invasive surgery (MIS) procedures became the golden standard over open surgeries: The long shafts characterizing laparoscopic tools prevent the surgeon from properly perceiving forces. Haptic distortion became conclusive when robotic MIS was introduced to enhance dexterity- and vision-related MIS issues. ${ }^{5}$ Multiple control modalities were proposed for robotic MIS: teleoperation frameworks to perform small-scale precise procedures ${ }^{6}$; autonomous systems to also foster repeatability ${ }^{7}$; and hands-on control, possibly complemented by virtual fixtures, to keep decisionmaking on the surgeon side for a safer approach. ${ }^{8}$ Teleoperated robotic MIS systems, in particular, eliminated direct haptic perception because of the physical separation between the master and the slave side. Any subsequent attempts to reintegrate force reflection capabilities had to face technical challenges, including a balance between system stability and transparency and the need to codevelop smart-sensing tools also compatible, eg, with sterilization and biocompatibility issues. 
After several years during which the lack of haptic feedback was considered a limitation for the $d V,{ }^{9}$ the mainstream opinion has become that robotic surgeons (necessarily trained on the $\mathrm{dV}$ ) can compensate by using visual feedback, so that they do not suffer from that shortage. ${ }^{10}$ On the other hand, many research studies claimed clear benefits from haptic feedback restoration for clinical applications/tasks requiring mechanical contrast perception for tissue discrimination (intraoperative palpation ${ }^{11-15}$ ), precise tool-tissue interaction force rendering (incision/ dissection, ${ }^{3,16-19}$ catheter steering, ${ }^{20}$ needle driving/suturing ${ }^{21-25}$ ), or tissue/organ safe manipulation (tissue clutching, organ retraction ${ }^{26,27}$ ). Furthermore, haptic information could also enrich the training phase of robotic surgeons. ${ }^{28-31}$ Not all the market players, however, shared the reluctance. For instance, the Sensei Robotic Catheter System (Hansen Medical, Mountain View, CA, USA), FDA cleared in 2007 and used for treating cardiac arrhythmia, is able to provide force feedback ${ }^{32}$ using a customized omega.medical haptic interface (Force Dimension, Nyon, Switzerland). Moreover, a novel system for laparoscopic surgery in the abdomen/pelvis of human adult has been FDA cleared earlier this year (2018), namely the Senhance Surgical System (TransEnterix Inc., Morrisville, NC, USA). Such a system, which features force sensing for multiple degrees of motion and force awareness for the entire length of the instrument during surgery, ${ }^{33}$ can be regarded to as a $d V$ competitor, in particular for gynecologic and colorectal procedures. Therefore, even the core market sectors seem to be questioning their pristine reluctance, and the claim that haptic feedback is not needed seems not to be monolithic.

Considering research literature, several studies tried to quantitatively assess the role of haptic feedback and to explore its potential applications in surgery. Intraoperative palpation to localize hard inclusions or vessels was identified as a key field of interest for force reflection. Indeed, visual feedback, which is still used to compensate, does not prevent from inaccurate discrimination whereas, eg, palpation of a phantom with stir straw inclusions in Gwilliam et al $^{14}$ showed that haptic feedback allowed for enhanced discrimination (in particular by experienced surgeons) and for reduced applied forces. Tissue incision was identified as an additional task of interest. In Wagner et al, ${ }^{3}$ force feedback was conveyed during a blunt dissection task: The authors guessed that perceiving the mechanical contrast along the dissection plane may serve to prevent users from exerting large forces. Indeed, the absence of force feedback increased the average force applied to the tissue by at least $50 \%$ and increased the peak force by at least a factor of 2. Complementarily, haptic feedback was shown to be beneficial for contactless incision as well, in particular for improving surgeon perception of the incision depth. In Fichera et al, ${ }^{16}$ information about laser incision depth, as estimated using laser parameters and irradiation time, was used to convey haptic sense to surgeons, who were asked to create ablation cavities with predetermined target depths. The results suggested that combined kinesthetic/vibrotactile feedback could increase accuracy compared with visual feedback, and both options increased accuracy compared with the working condition without feedback. Finally, soft tissue grasping represents an additional challenging task in robotic MIS. Tissue manipulation functional, eg, to suture tying becomes prone to damage in the absence of a proper rendering of the tool grasping force. ${ }^{34}$ Haptic guidance was thus proposed to improve dexterity, tool controllability, and tool-tissue interaction quality. In Moody et $\mathrm{al}^{22}$ the authors showed that haptic feedback allowed for a more accurate suture, while also reducing task completion time. Grasping, palpation, and incision thus emerged as relevant surgical tasks which could benefit from the introduction of force reflection to the master side.

However, the related results did not allow for a univocal assessment. For instance, in Fichera et al, ${ }^{16}$ haptic feedback led to improvements (in terms of reduced incision errors and variability) that, however, were not statistically significant. Moreover, inconsistent results were reported for task completion times. For instance, while in Moody et al, ${ }^{22}$ haptics led to a shortening, Demi et al ${ }^{19}$ reported significantly increased completion times for robotic MIS (either with or without haptics) compared with manual interventions, yet robotic MIS completion times with haptics did not differ from those without haptics in a statistically significant way (even if without haptics the users were approximately $10 \%$ faster). In addition, although several clinical studies supported the introduction of the aforementioned Senhance Surgical System, ${ }^{35}$ to the best of our knowledge, there are no publications comparing its performance when operated with and without haptic feedback. As a matter of fact and in spite of the resurgent interest, a conclusive statement on the potential benefits achieved by restoring haptic feedback in robotic MIS platforms seems still to be missing.

We tackled the aforementioned gap by leveraging the possibility to run grasping, palpation, and incision tasks on a single robotic platform, namely the da Vinci Research Kit (dVRK). We used the dVRK slave motor currents to estimate the forces to be reflected to the master, for ease of development, and to foster a unified working platform for all the considered tasks. As a substitutive master, we integrated into the dVRK a seven-DoF haptic interface, thus also allowing for force reflection during grasping. We designed a user study in close collaboration with medical doctors (robotic surgeons): We assessed the role of kinesthetic force feedback by performing clinically relevant tasks both with and without feedback. We accounted for user expertise by enrolling five expert surgeons, five medical residents, and five nonsurgeons, and we used task-specific quantitative performance metrics, complemented by a subjective evaluation administered to each user.

The rest of the paper is structured as follows. Section 2 mainly recalls the integration of the seven-DoF haptic interface into the dVRK and the user study design (user groups; rationale, protocol, and metrics for each task; data analysis). Section 3 reports taskspecific results and the complementary subjective assessment, which are discussed and integrated with concluding remarks in Section 4.

\section{2 | MATERIALS AND METHODS}

\section{1 | Master haptic device integration into the dVRK}

The dVRK is an "open-source mechatronic system" allowing to control retired first-generation da Vinci system instruments. ${ }^{36}$ It was devised 
as a shared platform for development by research groups worldwide, and it is currently available at 30 research sites. The dVRK working principle retraces that one of $\mathrm{dV}$, being based on a teleoperation framework between a master console consisting of two serial chain manipulators (master tool manipulators), directly handled by the surgeon, and two seven-DoF slave arms (patient-side manipulators). Task-specific, interchangeable endowrist tools are mounted on the patient-side manipulators. The dVRK architecture also allows for an additional endoscopic camera manipulator, which however was not included in the specific dVRK platform available to the authors. The dVRK software architecture is based on the open-source cisst libraries $^{37}$ and the Surgical Assistant Workstation (SAW) platform. The cisst libraries provide some base classes to implement thread-safe components that can be dynamically loaded and connected with each other at runtime. The default dVRK software stack relies on multiple components for input/output, low-level controller, arm control (homing, kinematics), and simple teleoperation, together with a console class to manage the whole system. The dVRK libraries also include Qt Widgets that can be reused across components, as well as Robot Operating System (ROS) components that convert messages between the dVRK core and ROS (both publishers and subscribers). ${ }^{38}$

We considered a sigma.7 haptic interface (Force Dimension, Nyon, Switzerland), available to the group in its right-handed configuration, as master for the dVRK. Its kinematics consists of a delta-based parallel structure, which supplies three translational DoFs to a wrist featuring three rotational DoFs and also integrating a one-DoF gripper. Handle rotations are thus decoupled from translations. All the seven DoFs are active, in particular the grasping DoF, which is not active in the dVRK master tool manipulators. Moreover, sigma.7 haptic transparency is fostered by gravity compensation, and a software development kit provides a set of real-time $C$ routines for advanced control (eg, to get/set positions and forces in Cartesian space). We physically connected the sigma.7 to the controller of one dVRK slave through a Linux PC also running the dVRK software interface. The slave arm was equipped with a Large Needle Driver Endowrist tool as endeffector. The sigma.7 encumbrance prevented a physical substitution at the dVRK master site, so that we renounced the dVRK binocular vision system in favor of an additional display showing the images recorded by a two dimensional (2D) high-resolution C920 HD PRO webcam (Logitech Europe, Lausanne, $\mathrm{CH}$ ). Consistently, we provided the user with the clutching function, originally available through the dVRK foot pedal tray, by means of a commercial usb foot switch (iKKEGOL, Shenzhen, China). The experimental platform is shown in Figure 1.

To integrate the sigma.7 with the existing dVRK software stack, we implemented two novel components. The first original contribution regards the master arm: It is a cisst SAW wrapper on top of the main header files (dhdc.h and drdc.h) of the sigma.7 software development kit. This new component has an interface similar to the dVRK master tool manipulator interface and can be dynamically loaded: It is possible to specify which master arm to use (either dVRK master tool manipulators or sigma.7) through the main dVRK JSON configuration file. Considering that this new component is used within the same executable, there is no added latency. Furthermore, such a new wrapper uses a dedicated thread, so that it can run at the refresh rate defined by the sigma.7. The second original contribution regards teleoperation. We created a new teleoperation component expressly enabling force/torque feedback to the sigma.7, to be loaded using the dVRK JSON configuration file in place of the default one provided with the dVRK stack. Figure 2 recalls the dVRK software architecture and highlights the new components created for integrating the sigma.7 master. Let us remark that for monitoring/recording the relevant data, we

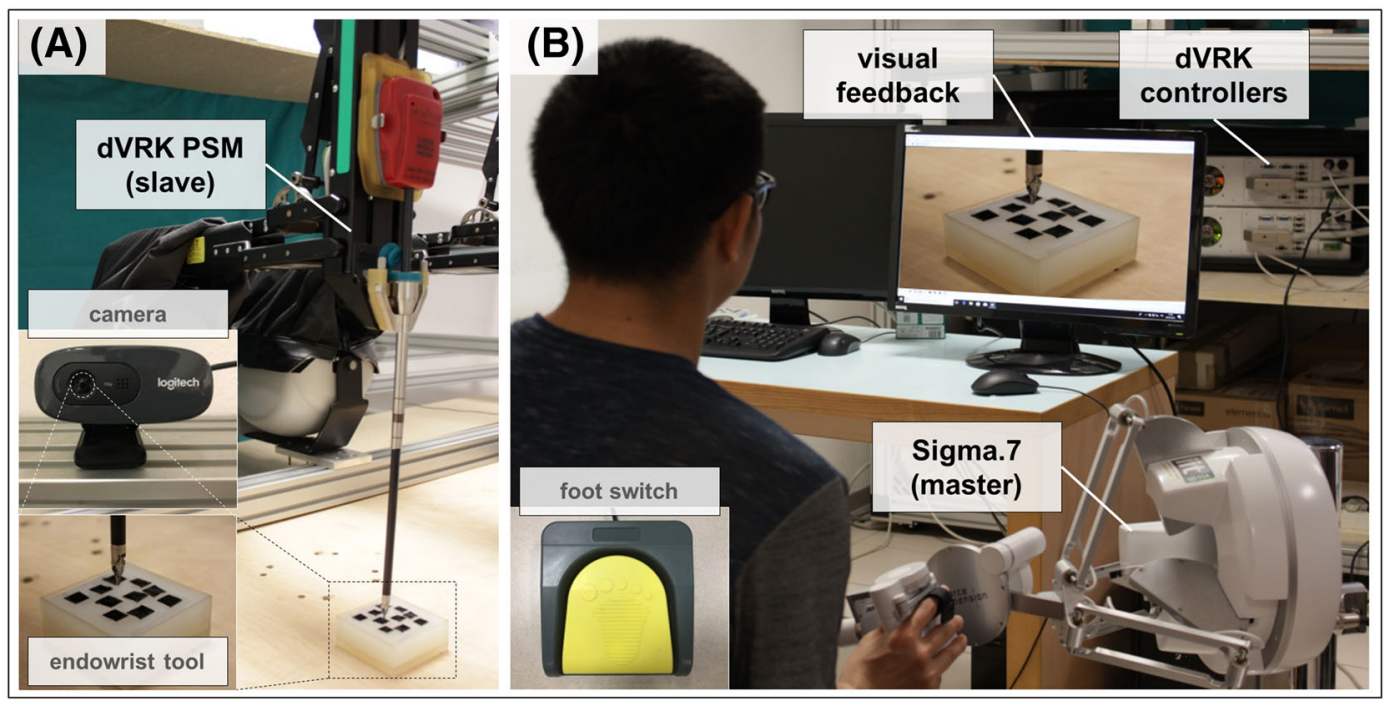

FIGURE 1 Experimental platform. A, A da Vinci Research Kit (dVRK) patient-side manipulator (PSM), equipped with a Large Needle Driver Endowrist tool, acts as slave arm. Top inset: camera. Bottom inset: endowrist tool close to the silicone phantom specifically used for palpation tests. B, At the master side, the user handles the sigma.7 haptic interface, which replaces a standard dVRK master tool manipulator (MTM). The haptic device allows the operator to guide the patient-side manipulator motion (feedforward) and to reflect a force to their hand/forearm (feedback). The force reflection also involves the grasping DoF, which is not active in the standard dVRK master tool manipulator. Inset: foot switch 


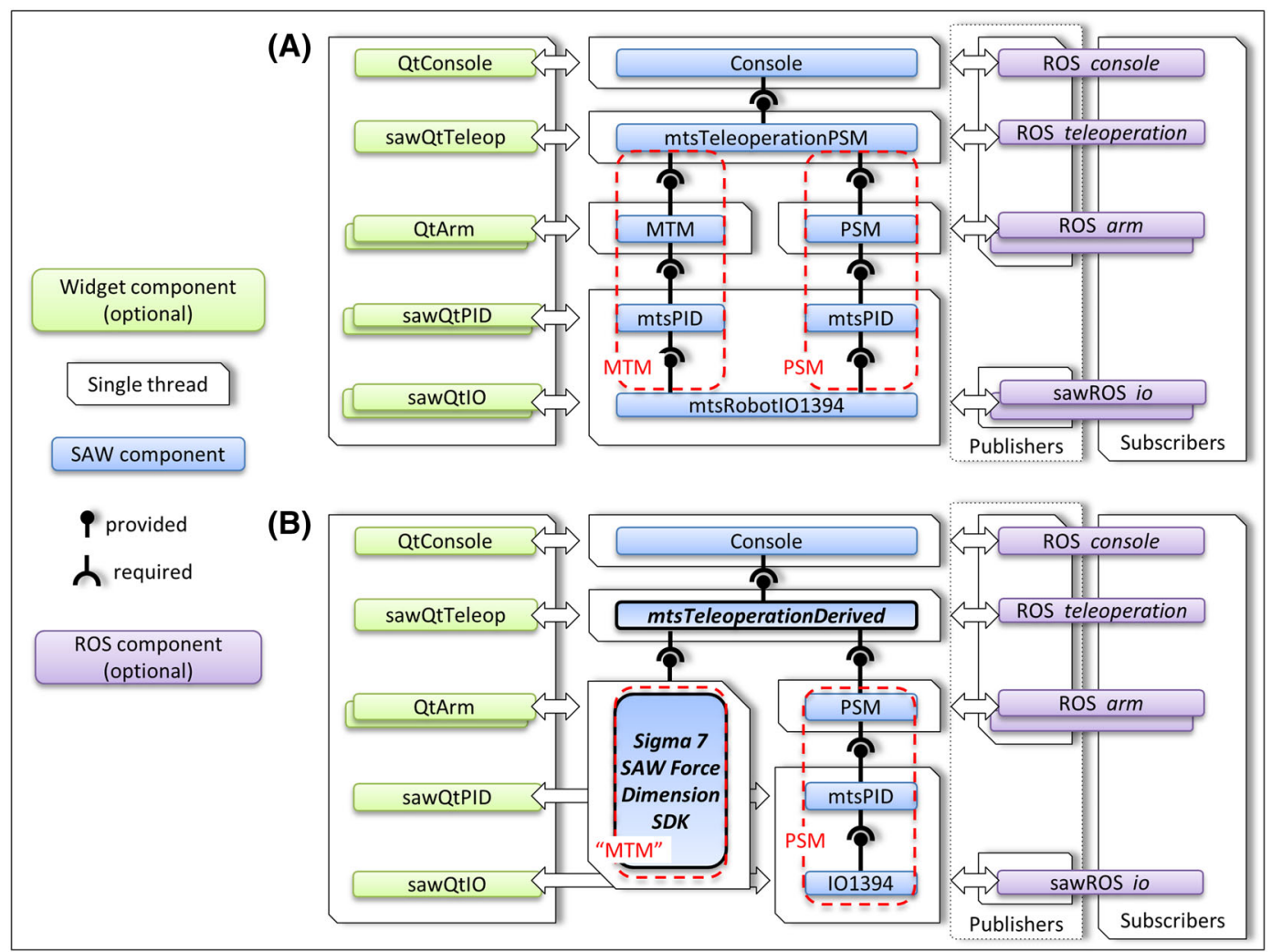

FIGURE 2 Software integration (schematic) of the experimental platform. A, Threads and components in the da Vinci Research Kit (dVRK) software stack. B, Components originally created for integrating the sigma.7 master in place of the dVRK master tool manipulator: (1) the sigma.7 SAW force dimension component replaces the arm ("MTM"), low-level controller ("mtsPID"), and input/output ("mtsRobotIO1394") components in the dVRK stack, and (2) the custom teleoperation component ("mtsTeleoperationDerived") is used in place of the default one ("mtsTeleoperationPSM")

could use all the ROS bridges already existing in the dVRK architecture, since both the newly created components are equivalent to the dVRK ones.

Considering the selected surgical tasks (ie, grasping, palpation, and incision; see Section 2.2.2), we only fed back forces (no torques) to the master, to also streamline the haptic feedback interpretation. As anticipated, we estimated the forces acting on the end-effector by using the dVRK slave motor currents. In particular, using motor specifications and gear ratios, the current feedback measured by the dVRK controllers can be converted to actuator efforts, whence joint efforts can be deduced through the coupling matrix between actuators and joints. Finally, by multiplying the joint efforts by the inverse of the Jacobian's transpose, we can estimate the wrench on the tool tip. Similarly, the actuator velocity estimation computed by the dVRK controller FPGA can be converted to a Cartesian velocity (twist). The involved parameter set is partially confidential, yet a subset of parameters (including, eg, gear ratio, encoder counts per turn, etc) is disclosed through a public generator aimed to create the JSON configuration file. ${ }^{39}$

The estimated forces were processed as follows. A movingaverage filter (500-point window size) was applied to the raw data. For palpation and incision, the filtered data were scaled to reflect $7.5 \mathrm{~N}$ on the master when applying $30 \mathrm{~N}$ to the slave. For grasping, the filtered data were scaled to reflect $1.2 \mathrm{~N}$ on the master when applying $0.12 \mathrm{~N}$ to the slave. The reference values at the master side (7.5 and $1.2 \mathrm{~N}$ ) were set by considering a safety margin for the sigma.7, while those at the slave side ( 30 and $0.12 \mathrm{~N}$ ) were defined through preliminary experiments, as estimated upper values for the corresponding tasks (see Section 2.2.2). Scaled data were sent to the master.

\section{2 | Experimental evaluation}

Based on literature, the potential of haptic feedback appears to be greater for procedures requiring accurate instrument positioning or modulation of tool/tissue contact forces or perception of the mechanical tissue contrast. Together with medical doctors and consistently with the use of a single master device, we thus identified three unimanual surgical tasks: grasping (test case (TC1), palpation (TC2), and incision (TC3). They are sketched in Figure 3, where the relevant corresponding forces on the end-effector and on the master handle are also shown.

Each test case was performed both with and without haptic feedback (whereas visual feedback was always available), and the sequence of such two modalities was randomized over subsequent users (who were informed on the specifically activated modality). For each test case and for each modality, three trials were performed, 


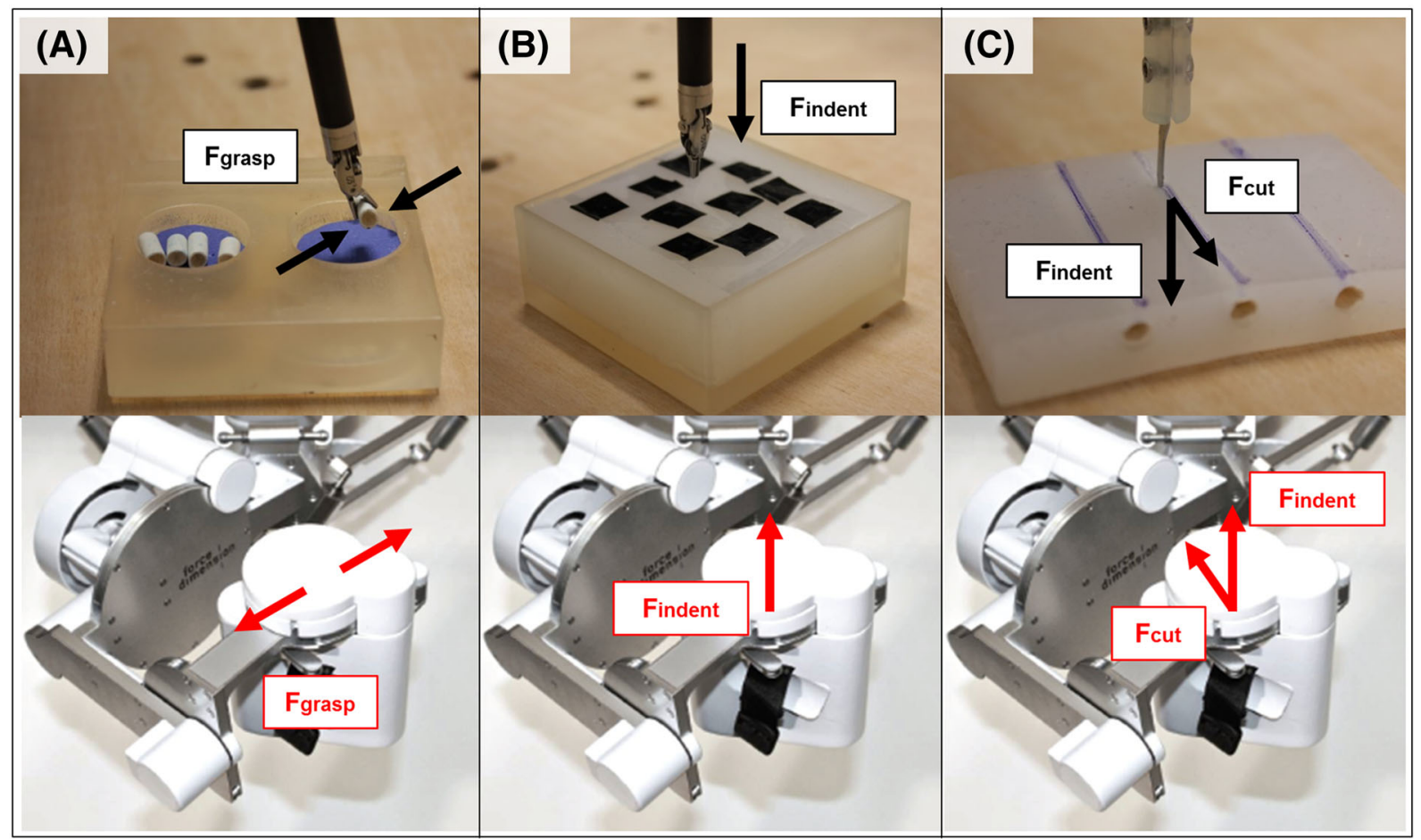

FIGURE 3 Experimental test cases. A, Grasping (TC1), here illustrated by pick-and-place of short cylindrical objects. B, Tissue palpation (TC2). C, Tissue incision (TC3). Black arrows indicate the relevant forces on the slave end-effector; red arrows indicate corresponding forces reflected to the sigma.7 master handle

thus leading to three repetitions. At the beginning of the experimental session, each user had the possibility to familiarize with the sigma.7 through its built-in demos, and by teleoperating the patient-side manipulator arm in free space for 5 minutes, thus understanding the kinematic mapping between master and slave, and the clutching function. In addition, before starting each task, users were provided with task-specific information, and they were allowed to train for 3 minutes (total time, with and without haptics).

\subsection{1 | Users}

We enrolled 15 right-handed-dominant users: 9 male and 6 female, average aged 32.3. They were classified into three groups: nonsurgeons (not a surgeon (NAS)), medical residents (nonexpert surgeons (NES)), and expert surgeons (ES). The experimental study design was balanced: five users per group. Specific demographics for each group are reported in Table 1. None of the users pointed out any deficiencies in visual/haptic perception abilities. The experimental protocol was approved by Scuola Superiore Sant'Anna Ethical

TABLE 1 User demographics per user group

\begin{tabular}{|c|c|c|c|}
\hline & $\begin{array}{l}\text { Not a Surgeon } \\
\text { (NAS) }\end{array}$ & $\begin{array}{l}\text { Nonexpert } \\
\text { Surgeon (NES) }\end{array}$ & $\begin{array}{l}\text { Expert } \\
\text { Surgeon (ES) }\end{array}$ \\
\hline No. of participants & 5 & 5 & 5 \\
\hline Age & $29.6 \pm 3.8$ & $31.4 \pm 2.2$ & $38.0 \pm 7.9$ \\
\hline Gender & 우: 3, ô: 2 & o $: 2$, ภ: 3 & $q: 1,8: 4$ \\
\hline
\end{tabular}

Committee, and all the participants provided written informed consent.

\subsection{2 | Test cases: rationale, protocol, and metrics}

\section{TC1: grasping}

Rationale From a clinical perspective, having grasping feedback allows the operating surgeon to feel and control the amount of grasping force used during organ displacement maneuvers. The ability to quantify these forces when applied to tender and subtle organs, such as small bowel, liver, or spleen, could be of fundamental importance to avoid iatrogenic damage.

Protocol We devised two subtasks: In both of them, the users directly exploited the patient-side manipulator grasper, and they were only fed back with the grasping force. The first subtask was a representative pick-and-place: The users were asked to move five small, deformable cylindrical objects from a starting well to an adjacent well (see Figure 3A), within maximum 2 minutes. That subtask was considered successful if all the five objects were correctly moved within the available time slot (unsuccessful otherwise). The second subtask was a representative vessel extraction: The users were asked to grasp and extract a vessel-like structure embedded in a silicone rubber matrix (like those visible in Figure 3A), which was previously exposed, within maximum 1 minute. That subtask, which is not detailed in Figure 3 for ease of representation, was considered successful if the vessel-like structure was fully extracted within the available time (unsuccessful otherwise). 
Metrics As figures of merit, we adopted the success/failure (ie whether the subtask was timely completed or not), the mean grasping force, and the peak grasping force.

\section{TC2: palpation}

Rationale Surgeons must often deal with neoplastic lesions in hollow viscus or lesions deeply located in solid organs, which in most cases are difficult to be detected. The lesions located in hollow viscus arise from the deep surface, and the surgeon is unable to detect them from the external layer, unless the lesion is large enough to reach that layer. In open surgery, such lesions are detected by means of palpation maneuvers. In robotic surgery, because of the absence of haptic feedback in the $d V$ system, surgeons are used to marking the neoplasia from the inside with a permanent endoscopic ink marker. Despite being very useful, that gesture may cause organ injuries (besides adding extra costs to an already expensive procedure). Moreover, the only way to detect lesions located in solid organs without the aid of haptic feedback is by exploiting laparoscopic/robotic ultrasound probes, which adds to the procedure complexity. Introducing haptic feedback in robotic MIS could overcome the aforementioned limitations.

Protocol The users were asked to indent a tissue-mimicking phantom in order to identify harder inclusions (simulating tumoral cores) embedded therein, without time constraints. Considering the different mechanical properties of human healthy and cancerous tissues, ${ }^{40}$ we used Ecoflex 00-30 silicone rubber (Smooth-On, Macungie, PA, USA) to mimic healthy tissue ${ }^{14}$ and Sylgard 184 PDMS (Dow Corning, MI, USA) to fabricate the inclusions. ${ }^{41}$ The palpation phantom was a $7 \times 7 \times 2 \mathrm{~cm}^{3}$ brick. Based on the indications of the 2003 American joint committee on cancer staging, according to which T1-stage tumors are sized $2 \mathrm{~cm}$ or less, ${ }^{42}$ we fabricated spherical inclusions having $1-\mathrm{cm}$ diameter. Moreover, dealing with a pilot study, we chose the same inclusion depth for all the inclusions, namely $1 \mathrm{~mm}$ below the phantom top surface. We marked with black adhesive tape 10 indentation sites on the phantom top surface (see Figure $3 \mathrm{~B}$ ), yet inclusions were only embedded underneath 6 of them. Inclusions were not visible because of both material opacity and covering tape. For each indentation site, the user was asked whether they thought to be probing the silicone matrix or a harder inclusion. The users exploited the patient-side manipulator end-effector tool tip to indent the phantom, and they were fed back with the indentation force. The phantom was randomly rotated (four possible orientations) by the investigator before each trial.

Metrics As figures of merit, we adopted the number of inclusion hits (max. 6), the mean indentation force, the peak indentation force, and the task completion time.

\section{TC3: incision}

Rationale While cutting soft tissues, surgeons systematically encounter embedded structures such as vessels and nerves. Leveraging the fact that these embedded structures are usually stiffer than the surrounding tissue matrix, the restoration of haptic feedback could permit to detect relevant anatomical landmarks, thus minimizing the critical risk of damage during incision.

Protocol The users were asked to perform an incision on a tissuemimicking phantom, along the direction of an embedded vessel-like inclusion, in order to expose it without causing damage, without time constraints. The phantom was a $10 \times 4 \times 2 \mathrm{~cm}^{3}$ brick. We used Ecoflex 00-30 silicone rubber (Smooth-On, Macungie, PA, USA) for the phantom matrix. The vessel-like inclusion was obtained by inserting a 4-cm-long stir straw, $2 \mathrm{~mm}$ below the phantom top surface. The vessel direction was highlighted by a corresponding dark line drawn on the top surface (see Figure $3 \mathrm{C}$ ), to provide users with some geometrical guidance while leaving their focus on the inclusion depth discrimination. We equipped the patient-side manipulator endeffector with a carbon steel surgical sterile blade (Swann Morton, Sheffield, UK), put on the end-effector jaws through a threedimensional (3D)-printed miniature adapter. The users were fed back with the indentation force (ie, the component perpendicular to the top phantom surface) and the cut force (ie, the component parallel to the vessel-like inclusion). The task was considered successful if the vessel-like structure was exposed without causing any damages such as cuts on the straw surface (unsuccessful otherwise).

Metrics As figures of merit, we adopted the success/failure (ie, whether the vessel was exposed without damage or not), the mean cutting force, the peak cutting force, the mean indentation force, the peak indentation force, and the task completion time.

\section{Complementary qualitative metrics}

At the end of the experimental session, each user was asked to fill in a questionnaire based on the System Usability Scale (SUS), ${ }^{43}$ combined with a Likert scale for assessing the scores. In particular, users were asked to separately assess the dVRK-sigma.7 platform with and without haptic feedback (the latter case being motivated by the light differences with respect to the standard dVRK configuration). The questionnaire is reported in Figure 4. The SUS global score was obtained by scaling to 100 the maximum score achievable through the nine administered questions, ie, 36. Such a score provided a complementary figure of merit reflecting the subjective perceptions of the users in performing the tasks with or without haptic feedback.

\subsection{3 | Data analysis}

The statistical analysis was carried out using MATLAB (The Mathworks, Natick, MA, USA) and SPSS (SPS srl, Bologna, Italy) software. The collected metrics were classified as either continuous or categorical variables, as reported in Table 2. The significance level was set to 0.05 .

As for continuous variables, we firstly performed the Shapiro-Wilk test for normality, followed by two-way ANOVA hypothesis test with a balanced design. The two relevant factors were the experimental condition (two levels: with or without force feedback) and the user expertise (three levels: NAS, NES, ES). Whenever a significant 


1. I think that I would like to use this
system frequently.
2. I found the system unnecessarily
complex.
3. I thought the system was easy to use.
4. I think that I would need the support of
a technical person to be able to use
this system.
5. I would imagine that most people
would learn to use this system very
quickly.
6. I felt very confident using the system.
7. I needed to learn a lot of things before
I could get going with this system.
8. I found the system very useful.
9. I perceive that the system actually
helped me in performing the tasks.

FIGURE 4 System usability scale (SUS)-derived questionnaire and Likert scale used for qualitatively assessing the effects of haptic feedback restoration, as perceived by the users

TABLE 2 Collected variables classification

\begin{tabular}{lll}
\hline Performance Metric & Categorical/Continuous & Test Case (TC) \\
\hline Success/failure & Categorical & TC1 $^{\mathrm{a}}, \mathrm{TC}^{\mathrm{b}}$ \\
\hline Grasping force & Continuous & TC1 \\
\hline No. of inclusion hits & Continuous & TC2 \\
\hline Indentation force & Continuous & TC2, TC3 \\
\hline Cutting force & Continuous & TC3 \\
\hline Task completion time & Continuous & TC2, TC3 \\
\hline
\end{tabular}

aSuccess: the (sub)task was timely completed (failure otherwise).

buccess: the task was completed without damaging the vessel-like structure (failure otherwise).

difference was found, post hoc Bonferroni correction was performed to identify between which groups that statistical significance lied and to eliminate potential false-positive results from the analysis.

As for categorical variables, we performed a three-way log-linear analysis. The three relevant factors were task success/failure, the experimental condition (two levels: with or without force feedback), and the user expertise (three levels: NAS, NES, ES).

\section{3 | RESULTS}

The results (mean \pm standard deviation) of the grasping, palpation, and incision test cases are reported in Figures 5, 6, and 7, respectively, whereas Figure 8 shows the results of the complementary qualitative assessment. Concerning the subplots appearing in the aforementioned figures, a thicker borderline highlights a metric for which the user expertise turned out to be statistically significant, while a yellow background highlights a metric for which the haptic feedback resulted statistically significant. The yellow-highlighted subplots thus contain key results for the present study.

\section{1 | TC1: grasping}

Figure 5 shows the results for both the pick-and-place subtask, labeled by the grasp1 subscript, and the vessel extraction subtask, labeled by the grasp2 subscript. The results obtained in each trial are shown, together with the cumulative results. All the relevant continuous variables passed the Shapiro-Wilk test for normality.

The first and forth rows in Figure 5 report the success/failure metric, namely whether the associated subtask was successfully completed or not. In both cases, the three-way log-linear analysis produced a final model that retained all the effects. The likelihood ratio of that model was $\chi^{2}(0)=0, P=1$. In both cases, the highest order parameter that resulted significant from our analysis was "user expertise $\times$ success/failure" $\left(\chi^{2}(2)=7.604, P=.022\right.$ for grasp1, $\chi^{2}$ $(2)=97.738, P=.021$ for grasp2). To break down those effects and understand in between which groups the significance lied, we considered the standardized residuals: It resulted that NAS users performed significantly worse than the other groups, for both subtasks.

The fifth and sixth rows in Figure 5 show the resulting mean and maximum grasping force for grasp2. Two-way ANOVA revealed a statistically significant difference in the user expertise factor for $F_{\text {grasp2 }}^{\text {mean }}\left(F_{84,2}=4.06, P=.021\right)$. Post hoc analysis with Bonferroni adjustment revealed that the significance lied between NAS and ES groups $(P=.02)$. Moreover, two-way ANOVA revealed a statistically significant difference in the user expertise factor for $F_{\text {grasp2 }}^{\max }\left(F_{84,2}=6.72, P=.002\right)$. Post hoc analysis with Bonferroni adjustment revealed that the significance lied between the NAS and the ES groups $(P=.02)$.

\section{2 | TC2: palpation}

Figure 6 shows the results obtained in each trial, together with the cumulative results. All the relevant continuous variables passed the Shapiro-Wilk test for normality. 

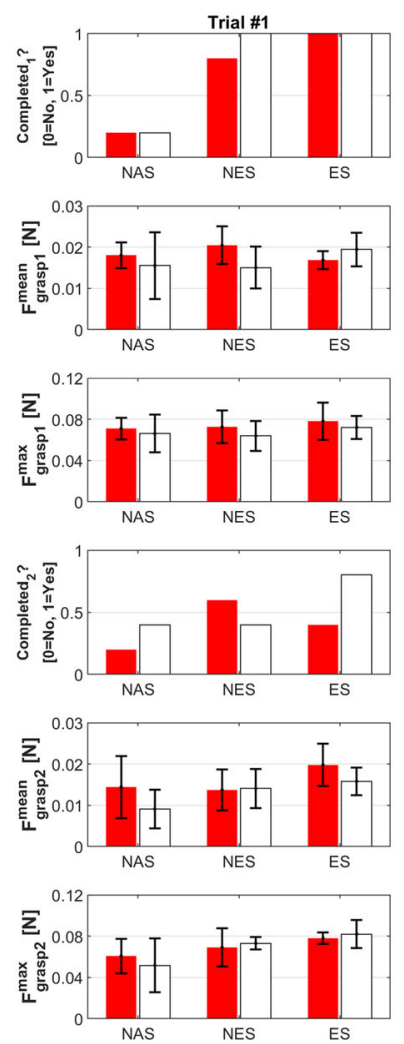
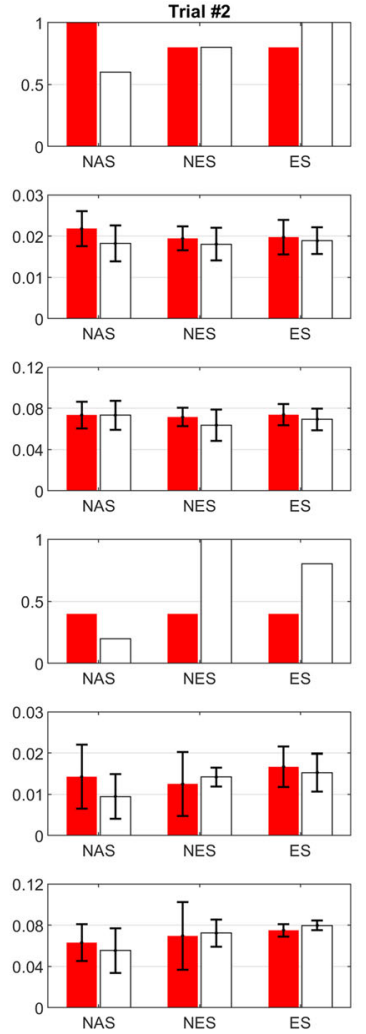
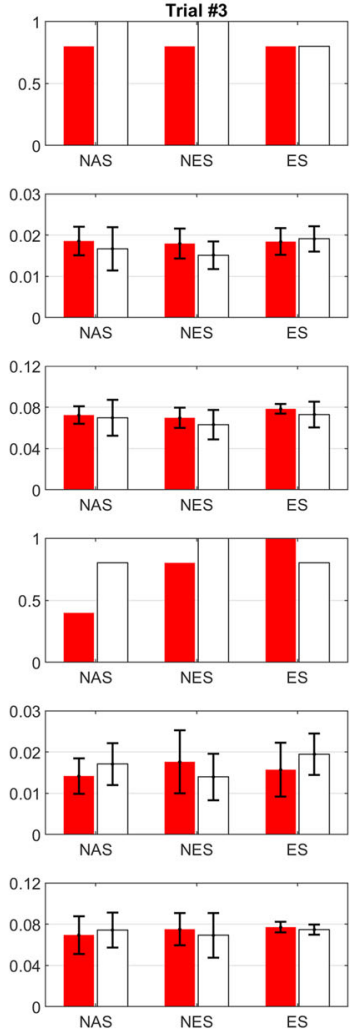
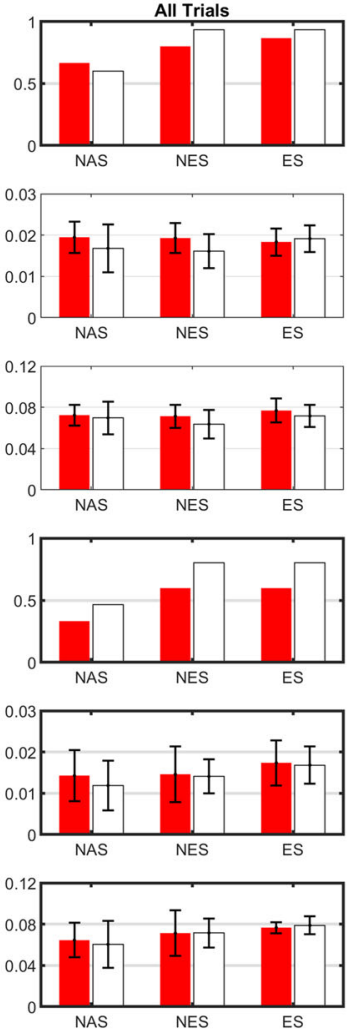

TC1 - Grasping (red: w/o haptics; white: w/ haptics)

FIGURE 5 Experimental results for the grasping test case. The first three columns report the results obtained in each trial, while the last column shows the cumulative results. Each row describes a specific performance metric; the first three rows refer to the pick-and-place subtask (labeled by subscripts grasp1 and 1), while the last three rows refer to the vessel extraction subtask (labeled by subscripts grasp2 and 2). In particular, the first and forth rows report the success/failure metric (ie, whether the associated subtask was successfully completed or not); the second and fifth rows report the mean grasping force; the third and sixth rows report the maximum grasping force. Subplots with thicker borderline highlight a metric for which the user expertise resulted statistically significant

The first row in Figure 6 reports the number of inclusion hits. Two-way ANOVA revealed a statistically significant difference in the experimental condition factor $\left(F_{84,1}=71.82, P<.0001\right)$. For completeness, let us report that no false positives were reported (possibly because the users could freely explore the phantom tissue, without time constraints).

The second row in Figure 6 reports the mean indentation force. Two-way ANOVA revealed a statistically significant difference in the user expertise factor $\left(F_{84,2}=4.14, P=.02\right)$. Post hoc analysis with Bonferroni adjustment revealed that the significance lied between the NAS and the NES groups $(P=.026)$.

The fourth row in Figure 6 reports the task completion time. Two-way ANOVA revealed a statistically significant difference in the experimental condition factor $\left(F_{84,1}=9.13, P=.003\right)$.

\section{3 | TC3: incision}

Figure 7 shows the results obtained in each trial, together with the cumulative results. All the relevant continuous variables passed the Shapiro-Wilk test for normality.
The first row in Figure 7 reports the success/failure metric, namely whether the vessel-like structure was exposed without damage or not. The three-way log-linear analysis produced a final model that retained all the effects. The likelihood ratio of that model was $\chi^{2}(0)=0, P=1$. The highest-order parameter that resulted significant from our analysis was "experimental condition $\times$ success/failure," $\chi^{2}(1)=9.772, P=.002$.

The third row in Figure 7 shows the maximum cutting force. Two-way ANOVA revealed a statistically significant difference in the user expertise factor $\left(F_{84,2}=4.23, P=.018\right.$ ). Post hoc analysis with Bonferroni adjustment revealed that the significance lied between the NAS and the NES groups $(P=.015)$.

The sixth row in Figure 7 reports the task completion time. Two-way ANOVA revealed a statistically significant difference in the user expertise factor $\left(F_{84,2}=6.69, P=.002\right)$. Post hoc analysis with Bonferroni adjustment revealed that the significance lied between the NAS group and both the NES $(P<.0001)$ and the ES groups $(P<.0001)$.

\subsection{Complementary qualitative metrics}

Figure 8 reports the SUS global score for both the NES and the ES groups. We only kept medical doctors because their opinion on 

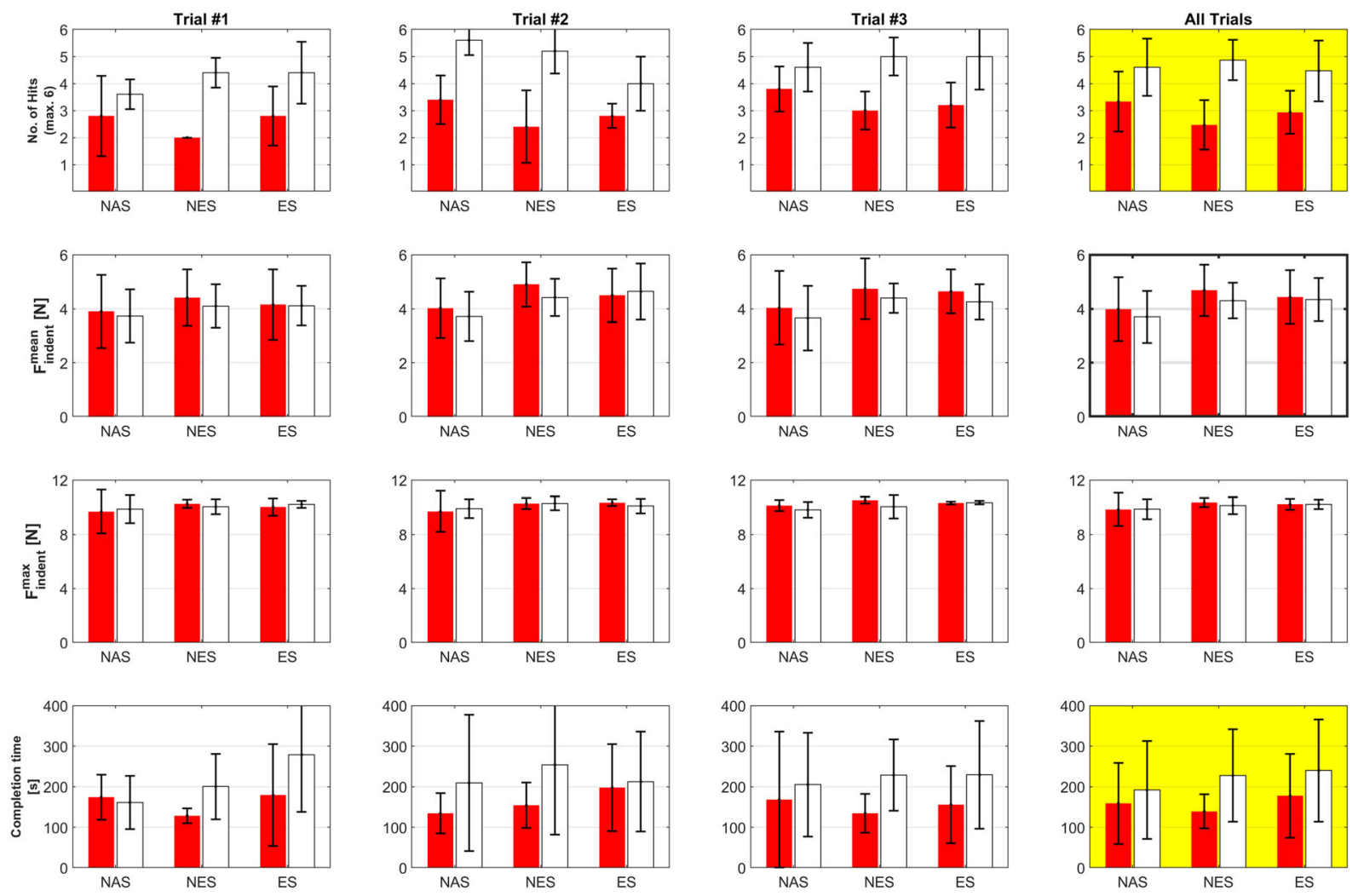

TC2 - Palpation (red: w/o haptics; white: w/ haptics)

FIGURE 6 Experimental results for the palpation test case. The first three columns report the results obtained in each trial, while the last column shows the cumulative results. Each row describes a specific performance metric. In particular, the first row reports the number of inclusion hits; the second and third rows show the mean and maximum indentation force, respectively; the fourth row reports the task completion time. Subplots with thicker borderline highlight a metric for which the user expertise resulted statistically significant. Subplots with yellow background highlight a metric for which the experimental condition, ie, the presence/absence of haptic feedback, turned out to be statistically significant

haptic feedback restoration is the most technically based and objective, thus relevant for our purposes (nonetheless, let us remark for completeness that the NAS results were consistent with the shown ones). Based on the SUS global score, no statistically significant difference was perceived between the two experimental conditions. However, based on extensive discussions with the involved medical doctors, it emerged that in performing the tasks, they clearly felt supported by the haptics, with direct connection to SUS Statement no. 9 ("I perceive that the system actually helped me in performing the tasks"), whence the related mark is also shown in Figure 8. Indeed, two-way ANOVA revealed a statistically significant difference in the experimental condition factor $\left(F_{1,16}=83.33\right.$, $P<.001$ ), explicitly highlighting a beneficial effect when restoring haptic feedback.

\section{4 | DISCUSSION}

We addressed the question of whether the lack of haptic feedback may have jeopardized the performance of current teleoperated robotic MIS platforms, in spite of the potential bias introduced by $\mathrm{dV}$ market supremacy on the mainstream opinion. To the purpose, we leveraged the closest research platform to $d V$, namely the dVRK: By taking advantage of its open software architecture, we integrated a master haptic device featuring seven active DoFs, namely the sigma.7. Moreover, we leveraged a unified platform to investigate three clinically relevant surgical tasks, ie, grasping, palpation, and incision. All the tasks, in terms of rationale, protocol, and performance metrics, were defined in close collaboration with medical doctors. Furthermore, we considered both the user expertise and the presence/absence of haptic feedback as experimental factors to be simultaneously considered.

We selected the sigma.7 master to also restore force feedback when grasping. However, the related quantitative results showed that force reflection did not impact significantly the user performance. This result, however, might have been affected by the relatively low involved forces. Indeed, when debriefing after the study was completed, the involved surgeons suggested to extend the grasping task by introducing an additional subtask devoted to ex vivo organ retraction. Indeed, that maneuver involves higher grasping forces during surgery, potentially harmful for tissue, so that surgeons must rely on visual cues such as color changing in vascularized regions in order to remain in a safe working domain. Differently, haptic force reflection made a statistically significant 

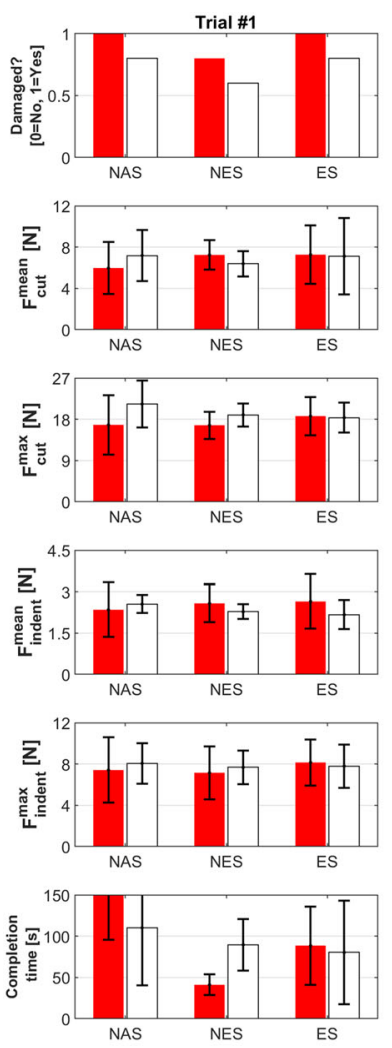
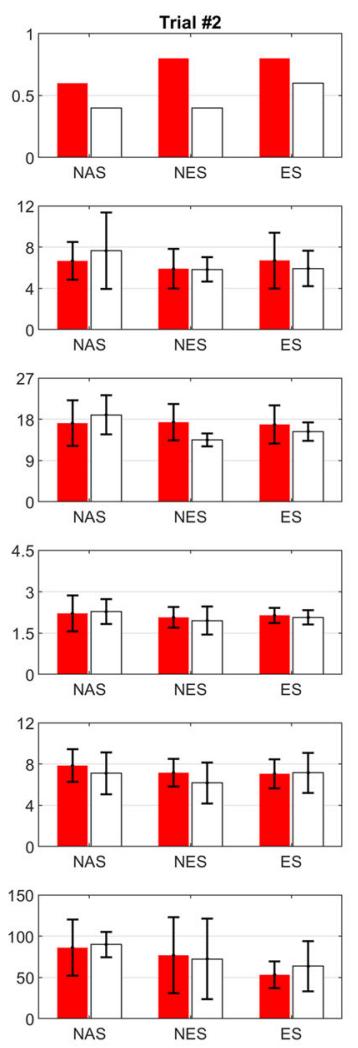
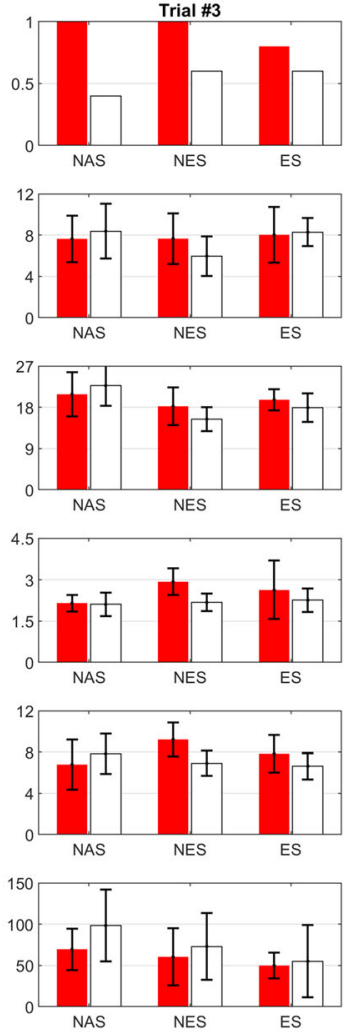
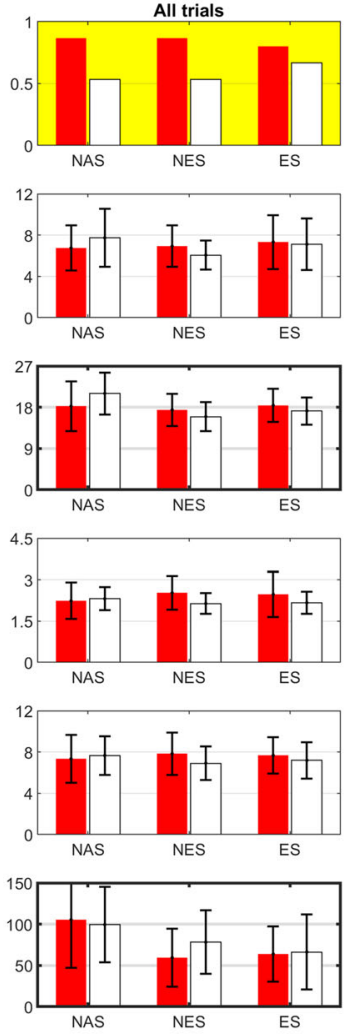

TC3 - Incision (red: w/o haptics; white: w/ haptics)

FIGURE 7 Experimental results for the incision test case. The first three columns report the results obtained in each trial, while the last column shows the cumulative results. Each row describes a specific performance metric. In particular, the first row reports the success/failure metric (ie, whether the vessel-like structure was exposed without damage or not); the second and third rows show the mean and maximum cutting force, respectively; the fourth and fifth rows show the mean and maximum indentation force, respectively; the sixth row reports the task completion time. Subplots with thicker borderline highlight a metric for which the user expertise resulted statistically significant. Subplots with yellow background highlight a metric for which the experimental condition, ie, the presence/absence of haptic feedback, turned out to be statistically significant

difference for palpation, both in terms of inclusion detection rate and inspection time. Incidentally, the recorded indentation forces were consistent with previous studies. ${ }^{44}$ Moreover and more importantly, it resulted that users took a longer time to palpate when provided with haptic feedback, yet they were able to detect more hard nodules. Similarly, haptic feedback also significantly reduced tissue damage rate during incision. Based on our findings, haptics can effectively improve key surgical outcomes for tasks requiring a relatively pronounced cognitive burden by the surgeon, possibly to be paid through longer completion times. This is consistent with previous reports ${ }^{19}$ and with the fact that, in the absence of force reflection, the users might have not spent time to interpret sensory perceptions at the master interface. Furthermore, the positive contribution of haptic feedback was also corroborated by the qualitative assessment gathered through the SUS-based questionnaire: Even if the global SUS score simply hinted a user tendency to prefer working with haptic feedback, that trend was statistically confirmed when focusing on the key statement 9 (users felt that haptic feedback was actively helping them in successfully completing the tasks). Based on further discussion following the experimental sessions, expert surgeons, in particular, expressed a strong appreciation for haptic force reflection during the incision task.

However, we are aware of the limitations affecting the present study. Primarily, we rendered the forces at the master handle based on the slave motor currents, by following an approach that complements the ones based, eg, on tool sensorization, master add-ons, or sensory substitution. ${ }^{45-48}$ Using current measurement for force estimation allows for seamless integration, and it is raising the interest of other researchers as well. ${ }^{49}$ Indeed, a current-based strategy might be favorable in view of certification, yet its accuracy for force feedback could be deteriorated by friction/stiction effects disturbing the derived signals. As a matter of fact, its superiority compared with the aforementioned alternatives should be better assessed, in terms of accuracy, complexity, sterilization/biocompatibility, and costeffectiveness. The latter aspect, in particular, was not tackled by the present study, and we are aware of the importance of economic factors for the effective deployment of surgical robotic technologies. In addition, based on the physical encumbrance of the sigma.7 device, we did not leverage the built-in dVRK binocular vision. This might have affected some results, in particular for ES who are used to 


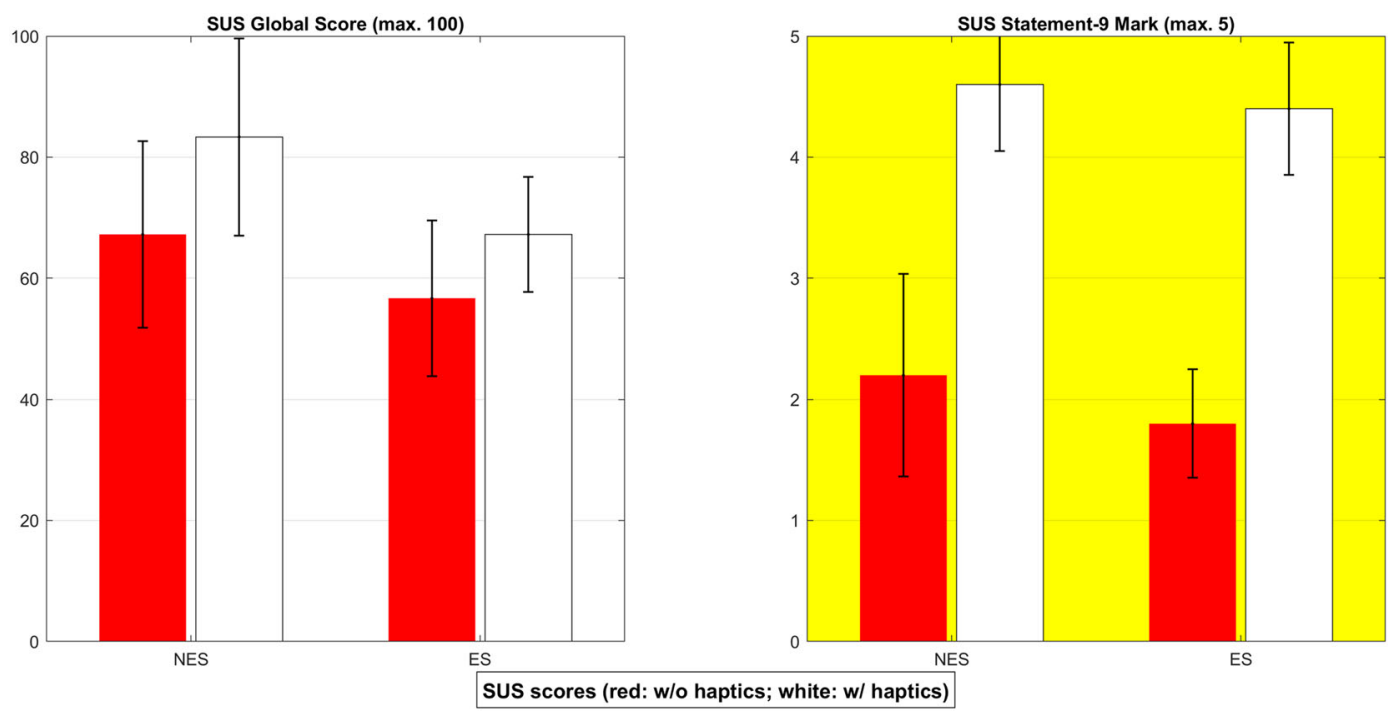

FIGURE 8 Results gathered through the SUS-based questionnaire. Only medical doctors (NES and ES) are considered, since their opinion is the most technically based and objective, thus relevant for our purposes. The SUS global score (left) refers to the whole questionnaire, while the SUS Statement-9 mark (right) specifically refers to the ninth statement ("I perceive that the system actually helped me in performing the tasks"), since it represents a key assessment point. Subplots with yellow background highlight a metric for which the experimental condition, ie, the presence/ absence of haptic feedback, turned out to be statistically significant

operating with $\mathrm{dV}$ immersive stereoscopic vision (whence, probably, their relatively lower SUS score compared with that one of NES). Hence, some future developments could also focus on reflecting the mechanical actions directly to the native dVRK masters, thus extending task space to bimanual procedures (yet renouncing to active feedback in grasping, since the related DoF is not active). However, for relevant tasks like knot tying, for which robotic surgeons struggle to detect the proper force intensity they are applying on the suture thread, ${ }^{25}$ the trade-off between bimanual operation and active grasping seems not to be trivial. In parallel, more extensive investigations on grasping with enhanced master devices like the sigma.7 would permit to understand the potential benefits of force feedback over a wider range of tasks, including the aforementioned retraction maneuvers. Indeed, the considered tasks may be oversimplified to the point that their results might not be sufficiently valid for more complex procedures. Additional parameter optimization would be valuable when targeting more complete and possibly clinically representative tasks. Furthermore, learning trends could be studied, even by focusing on specific tasks, by considering extended batches of trials, also periodically repeated over time. Finally, experiments involving ex vivo tissues should be also performed, still in close collaboration with medical doctors, to better assess the potential for clinical translation.

To conclude, by using the closest open platform to $\mathrm{dV}$, we showed that haptic feedback could effectively improve key surgical outcomes for tasks imposing a cognitive burden for the surgeon, possibly to be paid through longer completion times. Combining our results with the continuous interest in haptics by the research community and with the raising interest by competing commercial systems, ${ }^{35}$ the claim that haptic feedback in robotic MIS is unnecessary might be possibly revised, or at least more deeply investigated.

\section{ACKNOWLEDGMENTS}

The authors would like to thank Prof A.M. Sabatini and Dr A. Mannini (Scuola Superiore Sant'Anna) for scientific support related to the statistical analysis, Y. Huan (PhD student at Scuola Superiore Sant'Anna and Istituto Italiano di Tecnologia) for technical support related to the experimental setups, and all the involved users (University of Florence, Scuola Superiore Sant'Anna and Istituto Italiano di Tecnologia), for their time and dedication. This work was partly supported by Regione Toscana (PAR-FAS 2007-2013 programme) through the IMEROS project.

\section{ORCID}

Edoardo Sinibaldi (1D https://orcid.org/0000-0002-9755-3431

\section{REFERENCES}

1. Okamura AM. Haptic feedback in robot-assisted minimally invasive surgery. Curr Opin Urol. 2009;19(1):102-107.

2. Pacchierotti C, Meli L, Chinello F, Malvezzi M, Prattichizzo D. Cutaneous haptic feedback to ensure the stability of robotic teleoperation systems. Int J Robot Res. 2015;34(14):1773-1787.

3. Wagner CR, Stylopoulos N, Howe RD. The role of force feedback in surgery: analysis of blunt dissection. Proceedings of the 10th Symposium on Haptic Interfaces for Virtual Environment and Teleoperator Systems; 2002 March 24-25; Orlando, FL, USA. IEEE, 2002; 68-74.

4. Enayati N, De Momi E, Ferrigno G. Haptics in robot-assisted surgery: challenges and benefits. IEEE Rev Biomed Eng. 2016;9:49-65.

5. Camarillo DB, Krummel TM, Salisbury JK. Robotic technology in surgery: past, present, and future. Am J Surg. 2004;188(4):2-15.

6. Rosen J, Hannaford B, MacFarlane MP, et al. Force controlled and teleoperated endoscopic grasper for minimally invasive surgeryexperimental performance evaluation. v. 1999;46(10):1212-1221. 
7. Ayvali E, Ansari A, Wang L, Simaan N, Choset H. Utility-guided palpation for locating tissue abnormalities. IEEE Robotics Autom Lett. 2017;2(2):864-871.

8. Niccolini M, Castelli V, Diversi C, Kang B, Mussa F, Sinibaldi E. Development and preliminary assessment of a robotic platform for neuroendoscopy based on a lightweight robot. Int J Med Rob Comput Assisted Surg. 2016;12(1):4-17.

9. Freschi C, Ferrari V, Melfi F, Ferrari M, Mosca F, Cuschieri A. Technical review of the da Vinci surgical telemanipulator. Int J Med Rob Comput Assisted Surg. 2013;9(4):396-406.

10. Hagen ME, Meehan JJ, Inan I, Morel P. Visual clues act as a substitute for haptic feedback in robotic surgery. Surg Endosc. 2008;22(6): 1505-1508.

11. McKinley S, Garg A, Sen S, et al. A single-use haptic palpation probe for locating subcutaneous blood vessels in robot-assisted minimally invasive surgery. Proceedings of the 2015 IEEE International Conference on Automation Science and Engineering; 2015 August 24-28; Gothenburg, Sweden. IEEE, 2015; 1151-1158.

12. Brown JD, Ibrahim M, Chase EDZ, et al. Data-driven comparison of four cutaneous displays for pinching palpation in robotic surgery. Proceedings of Haptics Symposium'16; 2016, April 8-11; Philadelphia, PA, USA. IEEE, 2016; 147-154.

13. Hergenhan J, Rutschke J, Uhl M, et al. A haptic display for tactile and kinesthetic feedback in a CHAI 3D palpation training scenario. Proceedings of the 2015 IEEE Conference on Robotics and Biomimetics; 2015 December 6-9; Zhuhai, China. IEEE, 2015; 291-296.

14. Gwilliam JC, Mahvash M, Vagvolgyi B, et al. Effects of haptic and graphical force feedback on teleoperated palpation. Proceedings of the 2009 IEEE International Conference on Robotics and Automation; 2009 May 12-17; Kobe, Japan. IEEE, 2009; 677-682.

15. Li M, Faragasso A, Konstantinova J, et al. A novel tumor localization method using haptic palpation based on soft tissue probing data. Proceedings of the 2014 IEEE International Conference on Robotics and Automation; 2014 May31-June7; Hong Kong, China. IEEE, 2014; 4188-4193.

16. Fichera L, Pacchierotti C, Olivieri E, et al. Kinesthetic and vibrotactile haptic feedback improves the performance of laser microsurgery. Proceedings of Haptics Symposium'16; 2016, April 8-11; Philadelphia, PA, USA. IEEE, 2016; 59-64.

17. Luo J, Kania P, Banerjee PP, et al. A part-task haptic simulator for ophthalmic surgical training. Proceedings of the 2016 IEEE Symposium on 3D User Interfaces; 2016, March 19-20; Greenville, SC, USA. IEEE, 2016; 259-260.

18. Pyunghwa K, Soomin K, Young-Dai $P$, et al. Force modeling for incisions into various tissues with MRF haptic master. Smart Materials and Structures. 2016;25(3):035008. (13pp)

19. Demi B, Ortmaier T, Seibold U. The touch and feel in minimally invasive surgery. Proceedings of the 2005 IEEE International Workshop on Haptic Audio Visual Environments and their Applications; 2005 October 1-2; Ottawa, Ontario, Canada. IEEE, 2005; 33-38.

20. Guo J, Guo S, Yu Y. Design and characteristics evaluation of a novel teleoperated robotic catheterization system with force feedback for vascular interventional surgery. Biomed Microdevices. 2016;18(5):76. (16 pp)

21. Pacchierotti C, Abayazid M, Misra S, Prattichizzo D. Teleoperation of steerable flexible needles by combining kinesthetic and vibratory feedback. IEEE Trans Haptic. 2014;7(4):551-556.

22. Moody L, Baber C, Arvanitis TN. Objective surgical performance evaluation based on haptic feedback. Stud Health Technol Inform. 2002; 85:304-310
23. De Lorenzo D, Koseki Y, De Momi E, et al. Coaxial needle insertion assistant with enhanced force feedback. v. 2013;60(2):379-389.

24. Meli L, Pacchierotti C, Prattichizzo D. Experimental evaluation of magnified haptic feedback for robot-assisted needle insertion and palpation. Int J Med Rob Comput Assisted Surg. 2017;13(4):e1809. (14 pp)

25. Talasaz A, Trejos AL, Patel RV. The role of direct and visual force feedback in suturing using a 7-DOF dual-arm Teleoperated system. IEEE Trans Haptic. 2017;10(2):276-287.

26. Wottawa CR, Genovese B, Nowroozi BN, et al. Evaluating tactile feedback in robotic surgery for potential clinical application using an animal model. Surg Endosc. 2016;30(8):3198-3209.

27. King $\mathrm{CH}$, Culjat $\mathrm{MO}$, Franco $\mathrm{ML}$, et al. Tactile feedback induces reduced grasping force in robot-assisted surgery. IEEE Trans Haptic. 2009;2(2):103-110.

28. Brown JD, O'Brien CE, Leung SC, Dumon KR, Lee DI, Kuchenbecker $\mathrm{KJ}$. Using contact forces and robot arm accelerations to automatically rate surgeon skill at peg transfer. IEEE Trans Biomed Eng. 2017;64(9): 2263-2275.

29. Chi W, Rafii-Tari H, Payne $\mathrm{CJ}$, et al. A learning based training and skill assessment platform with haptic guidance for endovascular catheterization. Proceedings of the 2017 IEEE International Conference on Robotics and Automation; 2017 May29-June3, Singapore. IEEE, 2017; 2357-2363.

30. Brown JD, Fernandez JN, Cohen SP, et al. A wrist-squeezing forcefeedback system for robotic surgery training. Proceedings of the 2017 IEEE World Haptics Conference, 2017 June 6-9, Fürstenfeldbruck (Munich), Germany. IEEE, 2017; 107-112.

31. Bark K, Gomez ED, Rivera C, et al. Surgical instrument vibrations are a construct-valid measure of technical skill in robotic peg transfer and suturing tasks. Proceedings of the $5^{\text {th }}$ Hamlyn Symposium on Medical Robotics; 2012 July 1-2; London, UK; 50-51.

32. Sensei Robotic System [online]. Available from: http://hansenmedical. com/us/en/cardiac-arrhythmia/sensei-robotic-system/product-overview [Accessed: 2018/11/08].

33. Senhance Surgical System [online]. Available from: https://www. senhance.com/us/home [Accessed: 2018/11/08]

34. Bethea BT, Okamura AM, et al. Application of haptic feedback to robotic surgery. J Laparoendosc Adv Surg Tech A. 2004;14(3):191-195.

35. Spinelli A, David G, Gidaro S, et al. First experience in colorectal surgery with a new robotic platform with haptic feedback. Colorectal Dis. 2018;20(3):228-235.

36. Kazanzides $P$, Chen Z, Deguet A, et al. An open-source research kit for the da Vinci(R) surgical system. Proceedings of the 2014 IEEE International Conference on Robotics and Automation; 2014 May31-June7; Hong Kong, China. IEEE, 2014; 6434-6439.

37. Kapoor A, Deguet A, Kazanzides P. Software components and frameworks for medical robot control. Proceedings of the 2006 IEEE International Conference on Robotics and Automation; 2006 May 15-19; Orlando, FL, USA. IEEE, 2006; 3813-1818.

38. Chen Z, Deguet A, Taylor RH, et al. Software architecture of the da Vinci Research Kit. Proceedings of the 2017 First IEEE International Conference on Robotic Computing; 2017 April 10-12; Taichung, Taiwan. IEEE, 2017; 180-187.

39. Deguet A, Chen Z. sawIntuitiveResearchKit project, GitHub file, 2013 (available at: https://github.com/jhu-dvrk/sawIntuitiveResearchKit/ blob/master/share/configGenerator.m\#L90).

40. Mariappan YK, Glaser KJ, Ehman RL. Magnetic resonance elastography: a review. Clin Anat. 2010;23(5):497-511.

41. Ahn B, Kim Y, Kim J. New approach for abnormal tissue localization with robotic palpation and mechanical property characterization. 
Proceedings of the 2011 IEEE/RSJ International Conference on Intelligent Robots and Systems; 2011 September 25-30, San Francisco, CA, USA. IEEE, 2011; 4516-4521.

42. Woodward WA, Strom EA, Tucker SL, et al. Changes in the 2003 American joint committee on cancer staging for breast cancer dramatically affect stage-specific survival. J Clin Oncol. 2003;21(17): 3244-3248.

43. Brooke J. SUS-a 'quick and dirty' usability scale. Usability Evaluation in Industry. C. Press: 1996.

44. Konstantinova J, Cotugno G, Dasgupta P, Althoefer K, Nanayakkara T. Palpation force modulation strategies to identify hard regions in soft tissue organs. Plos One. 2017;12(2):e0171706. (24 pp)

45. Shahzada K, Yurkewich A, Xu R, et al. Sensorization of a surgical robotic instrument for force sensing. Proc of SPIE. 2016;9702:97020U.

46. McMahan W, Gewirtz J, Standish D, et al. Tool contact acceleration feedback for telerobotic surgery. IEEE Trans Haptic. 2011;4(3): 210-220.

47. Akinbiyi T, Reiley CE, Saha S, et al. Dynamic augmented reality for sensory substitution in robot-assisted surgical systems. Proceedings of the 28th IEEE EMBS Annual International Conference, 2006 Aug30-Sept3, New York City, USA. IEEE, 2006; 567-570.

48. Sang H, Monfaredi R, Wilson E, Fooladi H, Preciado D, Cleary K. A new surgical drill instrument with force sensing and force feedback for robotically assisted otologic surgery. ASME J Med Dev. 2017;11(3): 031009. (10 pp)

49. Tzemanaki A, Abeywardena S, Psomopoulou E, et al. Using current measurement to estimate palpation and grasping forces in robotassisted minimally invasive surgery. Proceedings of the $8^{\text {th }}$ Joint Workshop on New Technologies for Computer/Robot assisted Surgery; 2018 September 10-11; London, UK; 95-96.

How to cite this article: Saracino A, Deguet A, Staderini F, et al. Haptic feedback in the da Vinci Research Kit (dVRK): $A$ user study based on grasping, palpation, and incision tasks. Int J Med Robotics Comput Assist Surg. 2019;15:e1999. https://doi.org/10.1002/rcs.1999 